\title{
Musculoskeletal disorders following excessive smartphone usage - a review article
}

\begin{abstract}
Smartphone usage is growing exponentially in recent times. Smartphone usage requires users to be in awkward postures for long periods of time which increases the chances of musculoskeletal disorders. In this review article, we have tried to assess the musculoskeletal disorders occurring due to excessive smartphone usage. The results show increased muscle activity of the upper trapezius and the neck muscles along with an increase in the head tilting and head flexion. Frequent smartphone usage also leads to fatigue and pain in the hand. So in conclusion, it can be said that increased smartphone usage leads to pain and musculoskeletal disorders.
\end{abstract}

Keywords: smartphone, musculoskeletal, posture, hand, wrist
Volume 12 Issue 4 - 2020

Vikram Khanna,' Harish Chandra Khanna'
'Department of Orthopaedics, Ranjana Hospital, India
'Department of Internal Medicine, Ranjana Hospital, India

Correspondence: Vikram Khanna, Consultant, Department of Orthopaedics, Ranjana Hospital, I3,D-Road

Prayagraj, 21 1003, Uttar Pradesh, India, Tel 8787085 I40,

Email 86.khana@gmail.com

Received: August 10, 2020 | Published: August 28, 2020

\section{Introduction}

Smartphones has become an irreplaceable part of our day to day activity. It is not just used for communication purposes but also for gaming, browsing the internet among other things. The last decade has seen a massive increase in hours and frequency of smartphone usage. ${ }^{1,2}$ In 2012 there were more than 6 billion smartphones in the world. ${ }^{3} \mathrm{~A}$ study showed that smartphones were being used more than 20 hours per week for purposes like communication, texting, emailing, gaming, social networking; thereby making the people dependent on mobile phones for connecting and communicating. ${ }^{4}$ This dependency has led to musculoskeletal disorders in users. These conditions have increased even more since the COVID 19 pandemic which has led to social distancing and the increased usage of digital platforms like mobiles for communication amongst people. Therefore, doctors and other health care providers should be well aware of such issues that are being faced due to mobile usage. The smartphones are generally held below the eye level with one or both hands and the touch screen is operated with the index fingers and thumbs. ${ }^{5}$ So the posture which is attained is that of forward neck flexion mostly for long durations. ${ }^{5-8}$ This long duration of bad posture along with the increased use of the thumb and other fingers while playing games is responsible for the musculoskeletal problems occurring in the users..$^{6-8}$ The symptoms which are faced by the users generally include pain/discomfort in the neck, shoulder, elbow, arms, wrist, and fingers. ${ }^{1,5}$ There are some studies available that have assessed the effects of increased smartphone usage. The purpose of this study is to provide a systematic review of the same.

\section{Findings}

The findings of the studies on smartphone usage can be divided into the following categories.

\section{Head and neck movement}

Lee et al. ${ }^{9}$ in their study mentioned that the cervical flexion angle was more while texting as compared to other activities. Also, this angle increases while sitting as compared to standing. ${ }^{10}$ Another study observed that the neck flexion angle was more in cases with neck pain. ${ }^{11}$ While another study observed that the head tilt and the neck flexion angle was more in user using a smartphone as compared to those not using the phone. ${ }^{10}$ Jung et al..$^{12}$ in his study observed that with the increase in the smartphone usage the scapular index and the craniovertebral angle also increased as compared to the infrequent smartphone users.

\section{Pain}

Measurement of pain was done by the VAS (Visual Analogue Scale) scoring in 5 studies. ${ }^{13-17}$ Shin \& Kim ${ }^{13}$ realized in their study that the VAS score increased while using the smartphone on the desk and increased further when used on the lap. Inalet al. ${ }^{14}$ in their study concluded that the pain was comparable in infrequent users and nonusers, but increased in frequent users. Two studies ${ }^{15,16}$ concluded that the pain threshold of the upper trapezial muscles also decreased in frequent users. Lee et al. ${ }^{17}$ in their study found that the pain was more in users with single hand usage as compared to 2 hand usage. In users who frequently texted it was seen that the texting hand had more pain as compared to the other hand.

\section{Exertion and discomfort}

Discomfort and exertion were analyzed in 2 studies. ${ }^{18,19}$ The first study ${ }^{18}$ compared the use of large buttons and small buttons. The smaller buttons increased the exertion on the first digital interosseous but decreased the exertion on the abductor pollicislongus and brevis, the other study ${ }^{19}$ mentioned that the discomfort and exertion were more after texting especially in the symptomatic patients.

\section{Thumb - finger - hand performance}

4 studies assessed TFH performance..$^{14,18-20}$ Xiong et al. ${ }^{18}$ in their study indicated the use of small buttons led to shorter fatigue times. The speed of usage was slower in flexion/extension as compared to the adduction/abduction of the thumb. Inalet al. ${ }^{14}$ in their study found a weak negative correlation between the pinch strength and the smartphone addition score and the Duruoz hand index score. Eapenet al..$^{20}$ in their study found a significant reduction in the tip and lateral pinch grip strength of the patient with thumb pain while texting.

\section{EMG}

Four studies assessed the Electromyography for muscle activity while using smartphones. ${ }^{14,15,16,18}$ Xiong et al..$^{18}$ in their study found that 
using small buttons led to increased activity of First Dorsal Interossei and decreased activity of Abductor PollicisBrevis muscle. Kim et al. ${ }^{15}$ found that the median frequencies decreased in the brachioradialis muscle after smartphone usage. Lee et al. ${ }^{16}$ found that the Upper trapezial, Extensor PollicisLongus, and Abductor Pollicis were being used more in single-hand typing as compared to 2 hand typing. Xieet al. ${ }^{19}$ found that smartphone usage with a single hand led to more muscle activity in the forearm. Also, it was seen that in patients with neck pain there was increased muscle activity in the neck and shoulder due to increased muscle spasm.

\section{Tendon - nerve diameter}

Three studies evaluated the tendon and nerve thickness in symptomatic ${ }^{20}$ and asymptomatic ${ }^{14,17}$ patients. Eapenet al. ${ }^{20}$ found fluid in the tendons of thumb at wrist level in $19 \%$ and at thumb level in $2 \%$ of symptomatic patients. 2 studies showed that frequent smartphone users had significantly larger Flexor PollicisLongus tendons and median nerves. ${ }^{14,17}$

\section{Discussion}

This study assesses the effect of increased smartphone usage on various parts of the body. This review study shows that musculoskeletal symptoms are induced in the neck following smartphone usage. ${ }^{9-13,19}$ Smartphone usage causes increased activity of upper trapezius, erector spine, and neck extensor muscles. ${ }^{16,19}$ This study also shows that head tilting and neck flexion increases when smartphones are used and is further aggravated with increase in the duration of smartphone usage. ${ }^{9-13,19}$ It has also been observed that users with cervical pain use smartphones with a more flexed position of the neck which further worsens the neck posture and increases the symptoms, forming a vicious cycle. ${ }^{10,11}$ This can be explained by the alteration in the motor control of the neck due to excessive smartphone usage leading to greater neck flexion. ${ }^{21,22}$ The neck flexion changes with the way of holding the smartphone and the task at hand. ${ }^{5,9}$ Sitting posture causes a greater neck flexion angle as compared to standing., ${ }^{9,13}$ This is because while standing if the head-neck angle exceeds a certain value then it causes postural instability which does not allow the flexion of the neck to exceed a certain limit; this does not occur in sitting posture. ${ }^{23}$ For shoulder elbow and the forearm area, there was an increase in pain and a decrease in the threshold of pain with increased frequency of smartphone usage. . $^{15,16,19}$ The possible explanation is that with increase in muscle usage there is muscle fatigue and reduction in the pain threshold. ${ }^{24,25}$ When a smartphone is used for long durations this leads to microscopic muscle damage which further aggravates the condition. ${ }^{15,16,26}$

This study also shows that single-handed usage of phones led to more damage to the thumb movements and muscles. ${ }^{12,16,19}$ Two-handed use of the phone not only provides better cooperation between holding and performing tasks on the phone. ${ }^{27}$ This also led to less activity and lesser fatigue and pain in the hand and neck using the smartphone. ${ }^{12,16,19}$ This study also showed that the functionality of the thumb was decreased with the use of smaller buttons smartphones. ${ }^{14,20}$ This can be attributed to the lack of precision required in pressing big buttons as compared to small buttons. There are also changes in the tendon and nerve in the hand. With frequent use of the smartphones, the hand gets adjusted to the layout and the functionality of the hand also gets affected. This also leads to musculoskeletal problems in the hand especially the dominant hand. It also has a negative impact on the muscular and nervous tissue of the hand. ${ }^{28}$ This can also lead to increased pressure in the carpal tunnel leading to carpal tunnel syndrome. ${ }^{29}$

\section{Conclusion and recommendations}

a. Use of both hands while using smartphones

b. Hold the phone at the high level

c. Control the neck flexion angle

d. Reduction of frequency of usage

e. Take a break in between long usage

f. During the break not only move the hand and wrist but the cervical spine and the shoulder

g. Usage of phones with bigger buttons

\section{Acknowledgments}

None.

\section{Conflicts of interest}

The authors declare there are no conflicts of interest.

\section{Funding}

None.

\section{References}

1. Jonsson P, Johnson PW, Hagberg M, et al. Thumb joint movement and muscular activity during mobile phone texting-A methodological study. $J$ Electromyogr Kinesiol. 2011;21(2):363-370.

2. Goggin G. Cell phone culture: Mobile technology in everyday life. New York: Routledge; 2012.

3. International Telecommunication Union. Measuring the Information Society, 2012.

4. Madge C, Meek J, Wellens J, et al. Facebook, social integration and informal learning at university: It is more for socialising and talking to friends about work than for actually doing work. Learn Media Technol. 2009;34(2):141-155.

5. Berolo S, Wells RP, Amick BC. Musculoskeletal symptoms among mobile hand-held device users and their relationship to device use: A preliminary study in a Canadian university population. Appl Ergon. 2011;42(2):371378.

6. Gold JE, Driban JB, Yingling VR, et al. Characterization of posture and comfort in laptop users in non-desk settings. Appl Ergon. 2012;43(2):392399.

7. Maniwa H, Kotani K, Suzuki S, et al. Changes in posture of the upper extremity through the use of various sizes of tablets and characters. In Int Conf Human Interface and the Management of Information. Berlin, Heidelberg: Springer; 2013;89-96.

8. Bababekova Y, Rosenfield M, Hue JE, et al. Font size and viewing distance of handheld smart phones. Optom Vis Sci. 2011;88(7):795-797.

9. Lee S, Kang H, Shin G. Head flexion angle while using a smartphone. Ergonomics. 2015;58(2):220-226.

10. Guan X, Fan G, Wu X, et al. Photographic measurement of head and cervical posture when viewing mobile phone: A pilot study. Eur Spine $J$. 2015;24(12):2892-2898.

11. Kim MS. Influence of neck pain on cervical movement in the sagittal plane during smartphone use. J Phys Ther Sci. 2015;27(1):15.

12. Jung SI, Lee NK, Kang KW, et al. The effect of smartphone usage time on posture and respiratory function. J Phys Ther Sci. 2016;28(1):186-189. 
13. Shin H, Kim K. Effects of cervical flexion on the flexion-relaxation ratio during smartphone use. J Phys Ther Sci. 2014;26(12):1899.

14. Inal EE, Çetintürk A, Akgonül M, et al. Effects of smartphone overuse on hand function, pinch strength, and the median nerve. Muscle Nerve. 2015;52(2):183-188.

15. Kim GY, Ahn CS, Jeon HW, et al. Effects of the use of smartphones on pain and muscle fatigue in the upper extremity. $J$ Phys Ther Sci. 2012;24(12):1255-1258.

16. Lee M, Hong Y, Lee S, et al. The effects of smartphone use on upper extremity muscle activity and pain threshold. $J$ Phys Ther Sci. 2015;27(6):1743-1745.

17. Akkaya N, Dogu B, Ünlü Z, et al. Ultrasonographic evaluation of the flexor pollicis longus tendon in frequent mobile phone texters. Am J Phys Med Rehabil. 2015;94(6):444-448.

18. Xiong J, Muraki S. An ergonomics study of thumb movements on smartphone touch screen. Ergonomics. 2014;57(6):943-955.

19. Xie Y, Szeto GP, Dai J, et al. A comparison of muscle activity in using touchscreen smartphone among young people with and without chronic neck-shoulder pain. Ergonomics. 2016;59(1):61-72.

20. Eapen C, Kumar B, Bhat AK, et al. Extensor pollicis longus injury in addition to De Quervain's with text messaging on mobile phones. J Clin Diagn Res. 2014;8(11):LC01-LC04.

21. Szeto GP, Straker LM, O'Sullivan PB. A comparison of symptomatic and asymptomatic office workers performing monotonous keyboard work-1: Neck and shoulder muscle recruitment patterns. Man Ther. 2005;10(4):270-280.
22. Szeto GP, Straker LM, O’Sullivan PB. A comparison of symptomatic and asymptomatic office workers performing monotonous keyboard work-2: Neck and shoulder kinematics. Man Ther. 2005;10(4):281-291.

23. Buckley JG, Anand V, Scally A, et al. Does head extension and flexion increase postural instability in elderly subjects when visual information is kept constant? Gait Posture. 2005;21(1):59-64.

24. Enoka RM, Stuart DG. Neurobiology of muscle fatigue. J Appl Physiol. 1992;72(5):1631-1648.

25. Allen DG, Lannergren J, Westerblad H. Muscle cell function during prolonged activity: Cellular mechanisms of fatigue. Exp Physiol. 1995;80(4):497-528.

26. Haaland KY, Mutha PK, Rinehart JK, et al. Relationship between arm usage and instrumental activities of daily living after unilateral stroke. Arch Phys Med Rehabil. 2012;93(11):1957-1962.

27. Cook DB, O’Connor PJ, Eubanks SA, et al. Naturally occurring muscle pain during exercise: Assessment and experimental evidence. Med Sci Sports Exerc. 1997;29(8):999-1012.

28. Ko K, Kim HS, Woo JH. The study of muscle fatigue and risks of musculoskeletal system disorders from text inputting on a smartphone. $J$ Ergon Soc Korea. 2013;32(3):273-278.

29. Trudeau MB, Young JG, Jindrich DL, et al. Thumb motor performance varies with thumb and wrist posture during single-handed mobile phone use. J Biomech. 2012;45(14):2349-2354. 